9 - ORIGINAL ARTICLE ISCHEMIA REPERFUSION

\title{
Protective perioperative strategy using a third generation hydroxyethyl starch during surgery in a murine model of liver reperfusion injury ${ }^{1}$
}

\author{
Estratégia protetora perioperatória usando um hidroxietilamido de terceira geração para \\ expansão volêmica durante a cirurgia em modelo murino de lesão de reperfusão hepática
}

\author{
Dora CatréI, Maria Francelina Lopes ${ }^{\mathrm{II}}$, Celeste Bento ${ }^{\mathrm{III}}$, António Silvério Cabrita ${ }^{\mathrm{IV}}$
}

\begin{abstract}
'MSc, Anesthesiologist, Anesthesiology Department, Sao Teotonio's Hospital, EPE, Viseu, Portugal. Responsible for design, intellectual and scientific content and manuscript writing.

IIPhD, Assistant Professor, Experimental Surgery, Faculty of Medicine, University of Coimbra. Pediatric Surgeon, Pediatric Hospital of the Coimbra's University Hospital Center, EPE, Coimbra, Portugal. Supervised all phases of the study and manuscript writing.

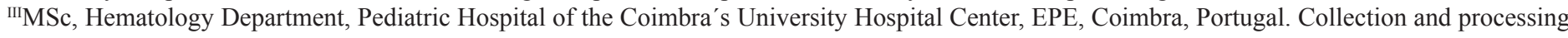
of study informations.

${ }^{\mathrm{IV}} \mathrm{PhD}$, Assistant Professor, Experimental Pathology, Faculty of Medicine, University of Coimbra, Portugal. Supervised the histopathological analysis.
\end{abstract}

\begin{abstract}
PURPOSE: To investigate whether a third generation colloid, hydroxyethyl starch (HES 130/0.4), used for perioperative fluid therapy, protects the rat liver against the late-phase response of ischemia/reperfusion injury (IRI) and if inhibition of neutrophil hepatic infiltration plays a part in this mechanism.

METHODS: Wistar rats were used ( 8 in each group). Three groups had IRI induced by lobar vascular occlusion (60 minutes) and reperfusion (24 hours) and received HES (13 mL/kg iv), 7.5\% saline (HS) $(13 \mathrm{~mL} / \mathrm{kg}$ iv) or no fluid. Three other groups were shamoperated and received the same fluid as the test groups. After 24 hours of reperfusion, blood was drawn for alanine aminotransferase (ALT) quantification and ischemic liver samples were taken for histological study (hematoxylin and eosin and chloroacetate staining of neutrophils).
\end{abstract}

RESULTS: HES treatment attenuated the elevation in serum ALT $(P=0.001)$ and reduced the extent of hepatocellular necrosis $(P<0.01)$ compared with the IRI controls. HES-mediated cytoprotection was associated with a decrease of infiltration of neutrophils in the necrotic areas $(P<0.05)$ compared with the untreated IRI rats, but not with the volume control IRI rats $(P>0.05)$.

CONCLUSION: Hydroxyethyl starch suppresses inflammatory response and ameliorates the late-phase response of hepatic ischemia/ reperfusion injury.

Keywords: Hetastarch. Ischemia. Reperfusion. Rats.

\section{RESUMO}

OBJETIVO: Investigar se um colóide de terceira geração (HES 130/0.4), utilizado para fluidoterapia perioperatória, protege o fígado de rato contra a resposta da fase tardia de isquemia/reperfusão e se a inibição da infiltração hepática de neutrófilos desempenha um papel neste mecanismo.

MÉTODOS: Foram utilizados ratos Wistar (8 em cada grupo). Três grupos tiveram lesão de isquemia/reperfusão (IRI) induzida por oclusão vascular lobar (60 minutos) e reperfusão (24 horas) e receberam HES (13 ml / kg iv), soro fisiológico a 7,5\% (HS) (13 ml / kg iv) ou nenhum fluido. Três outros grupos foram sham-operados e receberam o mesmo tipo de fluido dos grupos de teste. Após 24 horas de reperfusão, o sangue foi retirado para quantificação da alanina aminotransferase (ALT) e amostras de fígado isquêmico foram retiradas para estudo histológico (hematoxilina e eosina e coloração cloroacetato para neutrófilos).

RESULTADOS: O tratamento com HES atenuou a elevação da ALT sérica $(P=0,001)$ e reduziu a extensão da necrose hepatocelular $(P<0,01)$ em comparação com os controles da IRI. A citoproteção mediada por HES foi associada a uma diminuição da infiltração de neutrófilos nas áreas de necrose $(P<0,05)$ em comparação com os ratos não tratados IRI, mas não com os ratos controlo IRI $(P>0,05)$. CONCLUSÃO: HES suprime a resposta inflamatória e melhora a resposta na fase tardia da isquemia/reperfusão hepática.

Descritores: Hetamido. Isquemia. Reperfusão. Ratos. 


\section{Introduction}

Ischemia/reperfusion injury (IRI) to the liver is commonly encountered in clinical situations such as hemorrhagic shock, septic shock, abdominal surgeries, major hepatectomy and liver transplantation, especially if surgery is performed in patients with severe liver disease ${ }^{1,2}$. In these settings, substantial hepatocellular damage occurs during restoration of hepatic blood flow after a period in which circulation is extensively disrupted. Following the hypoxemic injury in the ischemic period, the subsequent reintroduction of oxygen into the liver leads to an aggressive inflammatory response. This occurs in two phases: the acute injury phase (early-phase), which is characterized by liver injury occurring within 1-6 hours after reperfusion, is associated with Kupffer cell activation, release of the pro-inflammatory cytokines, and generation of reactive oxygen species; the subacute-phase response (late-phase), which extends from 6 up to 24 hours of reperfusion in the rat model, is characterized by an extensive neutrophil infiltration and further production of the inflammatory mediators $^{2}$

Intravascular fluid therapy remains an important issue in liver surgery, where ischemia/reperfusion injury continues to be a major complication. The processes initiated during hepatic ischemia/reperfusion lead to liver dysfunction and remote organ injury, which may conduct to multiple organ failure and culminate in death. In this arena, fluids with known anti-inflammatory properties have become a major research interest.

Hydroxyethyl starch (HES) 130/0.4, a natural polymer of amylopectin, belongs to the group of starches that have been used as an intravascular volume expander during surgery. In addition to the intravascular volume expansion properties, provided by their oncotic characteristics and slow clearance rate from the circulation, these colloids also have a variable influence on other factors, such as coagulation, renal function and inflammation. The third generation product, HES 130/0.4, has been associated with safer clinical profiles on coagulation and renal function ${ }^{3-5}$. While reports suggest that intravenous administration of starches in general yields anti-inflammatory properties, both in experimental models and in clinical settings ${ }^{6-8}$, this merit has not yet been sufficiently addressed for the third generation products, especially in the late-phase of hepatic IRI.

By using an appropriate liver IRI rat model, the present study was designed to test the hypothesis that HES 130/0.4 has a protective effect in the late-phase of liver IRI ( 24 hours of reperfusion) and that this protection is associated with antiinflammatory properties.

\section{Methods}

Animals and experimental design

Adult male Wistar rats (Charles River Laboratories, L'Arbresle Cedex, France) weighing 225-325 g were housed in a temperature-controlled room on a 12 hours light/dark cycle and with access to normal rat chow and drinking water ad libitum. The study was approved by the Portuguese Authority responsible for the protection of animals used for experimental purpose (DSSPA, $n^{\circ}$ 0420/000/000/2008) and meets applicable European legislation and the standards of the National Institutes of Health, as set forth in the Guide for the Care and Use of Laboratory Animals (National Research Council, Washington: National Academy Press, revised 1996).

To explore the role of HES on the late-phase of hepatic ischemia/reperfusion injury (IRI), rats were randomized into six groups ( $n=8$, each). Liver IRI was induced in 3 groups (IRI+HES, IRI+HS and IRI-only) with 1 hour of segmental hepatic ischemia followed by 24 hours of reperfusion; two of these groups received either HES (IRI+HES) or $7.5 \%$ saline solution (IRI+HS), and the third group (IRI-only) was untreated and served as control. Three other groups were sham-operated (Sh-only, Sh+HES and Sh+HS) and served as normal controls. Group Sh-only had surgical manipulation only and the other two sham-operated groups received the same fluid as the test groups. Staff delivered the HES or $7.5 \%$ saline solutions blindly in similar unidentified syringes. They injected the solutions intravenously at the onset of reperfusion at a volume of $13 \mathrm{~mL} / \mathrm{kg}$ body weight. Mortality was registered. Animals were humanely killed after 24 hours of reperfusion and blood was drawn for serum alanine aminotransferase (ALT) quantification and ischemic liver samples were taken for histological study by light microscopy (hematoxylin and eosin and chloroacetate staining of neutrophils). Codes were used to mask the groups in ALT serum determinations and histopathological samples

\section{Rat model of hepatic ischemia/reperfusion injury}

We selected a non-lethal model of segmental (70\%) hepatic ischemia to allow prevention of mesenteric congestion. All surgical procedures were performed on intramuscular ketamine (115 $\mathrm{mg} / \mathrm{kg}$ ) and chlorpromazine (3.5 mg/kg) anesthetized animals, with body temperatures maintained at $37^{\circ} \mathrm{C}$. A midline abdominal incision was performed to enter the peritoneal cavity. In groups which included IRI induction, a microvascular clamp (TKL-2, Biover AG, Hergiswil, Switzerland) was placed across the vascular structures common to the left and median hepatic lobes 
for 60 minutes. To confirm its correct placement, ischemic color change was inspected. After that period the clip was removed. Immediately before reperfusion in the IRI +HES and IRI+HS groups, staff blindly administered $1 \mathrm{~mL}$ of the respective test solution into the left femoral vein in slow bolus and slowly infused the remaining volume (approximately $2 \mathrm{~mL} / \mathrm{min}$ ) after removing the clip. The animals in the IRI-only group did not receive any intravascular solution.

Animals of the Sh-only group underwent the same surgical procedure with the exception of vascular clamping and fluid injection. Animals in the $\mathrm{Sh}+\mathrm{HES}$ or $\mathrm{Sh}+\mathrm{HS}$ groups underwent a similar sham procedure (without IRI), and a volume of either $13 \mathrm{~mL} / \mathrm{kg}$ of HES or $7.5 \%$ saline solution, respectively, was injected into the femoral vein.

At the defined end-point ( 24 hours of reperfusion), the rats were re-anesthetized and blood was drawn from the aorta for ALT quantification and ischemic liver tissue samples were taken for subsequent histomorphological study. The surviving animals were killed by exsanguination.

\section{Serum alanine aminotransferase levels}

Serum ALT levels were evaluated based on the following protocol: blood samples were centrifuged to separate the plasma. Plasma samples were stored at $-20^{\circ} \mathrm{C}$ until use for ALT assay. ALT levels, expressed in international units per liter (IU/L), were determined using a standard dry chemistry system (Johnson \& Johnson Vitros 250; Ortho-Clinical Diagnostic, Inc., Rochester NY, USA).

\section{Histological examination and photoimage analysis}

At necropsy, liver samples from all rats were collected for histopathologic evaluation. Tissues were fixed in $10 \%$ formalin, embedded in paraffin and were then processed to obtain 5-micron thick paraffin sections. Sections were stained with hematoxylin and eosin and were also subjected to staining with the Naphthol AS-D Chloroacetate Esterase Kit (Sigma, St. Louis, MO). This technique, which was performed according to the manufacturer's instructions with modifications, allows the determination of chloroacetate esterase activity, which reflects neutrophil infiltration. Briefly, CAF-solution $(2.5 \mathrm{~mL}$ citrate +6.5 $\mathrm{mL}$ acetone $+0.8 \mathrm{~mL} 37 \%$ formalin) was added to deparaffinised in xylene and rehydrated in a graded alcohol series sections, for exactly 30 seconds, followed by rinse with water. Subsequently, $125 \mu \mathrm{L}$ of sodium nitrite solution $(0.1 \mathrm{~mol} / \mathrm{L})$ was added to 125 $\mu \mathrm{L}$ of Fast Red Violet LB Base solution and allowed to stand for 2 minutes. This solution was then added to $5 \mathrm{~mL}$ pre-warmed $\left(37^{\circ} \mathrm{C}\right)$ deionized water, $625 \mu \mathrm{L}$ of Trizmal TM 6.3 concentrate and 125 $\mu \mathrm{L}$ of Naphthol AS-D Chloroacetate solution. This solution was gently added to the tissue and the slides were then placed on an immunohistochemistry tray with dampened paper towel in the bottom and incubated for 3 hours at $37^{\circ} \mathrm{C}$, according to the protocol provided by the University of Kansas Medical Center (Margitta Lebofsky, Pharmacology Department), and then rinsed thoroughly in deionized water. Hematoxylin Solution Gill No. 3 was used for counter stain (1 minute). After rinsing with water, slides were mounted using aqueous mounting media. Sites of activity were identified by bright red granulation.

All slides were coded and examined by light microscopy to assess hepatocellular necrosis and the accumulation of neutrophils in hepatic tissue. This was performed by an experienced pathologist, blinded to the experimental groups, who performed a qualitative and quantitative analysis. Foci of extensive nuclear pycknosis (apoptosis), cytoplasmic hypereosinophilia, cell ballooning, loss of intercellular borders or with disintegration of hepatic cords (necrosis) and neutrophil infiltration were considered areas of relevant hepatocellular damage and were quantified using photoimage analysis. To do so, digital images of the stained slides were captured (Nikon Coolscope, Digital Microscope, Emílio de Azevedo Campos, S.A. Porto, Portugal) and necrotic liver areas and neutrophils, a correlate of tissue injury, were quantified using National Institutes of Health 1.42q ImageJ software. Areas of hepatocyte necrosis were delineated using the image analysis software and the degree of injury was expressed as the percentage of damaged liver architecture, measured in 10 randomly selected low-power fields (x5) per section; neutrophils, identified by staining and morphology, were counted in 10 high-power fields $(\times 40)$ per section under light microscopy.

\section{Statistical analysis}

Statistical analysis was performed using SPSS statistical software package (vs. 11.5, SPSS, Inc., Chicago, IL). Normality of distribution was determined using Kolmogorov-Smirnov and Shapiro-Wilk tests. Comparisons between multiple groups were performed with one-way ANOVA followed by Least Significant Difference test (normally distributed data). If the data were not normally distributed, the Kruskal-Wallis test (nonparametric ANOVA) was used, and, where differences were identified, pairwise comparisons were performed using Mann-Whitney U test with appropriate correction by Holm-Bonferroni method. Chi square test with Fisher's Exact test was used for categorical data. All differences were considered significant at $P<0.05$. Values are expressed as means \pm standard deviation (SD) and as median and 
interquartile range (IQR), as appropriate, and $n$ is the number of rats in each group.

\section{Results}

Effect of intravenous (iv) HES fluid on hepatic function

Hepatocellular damage was assessed by the serum ALT levels. The sham-operated rats showed similar values of ALT [median (IQR); Sh-only: 59.0 IU/L (38.0 IU/L); Sh+HES: 53.5 IU/L (26.5 IU/L); Sh+ HS 123.0 IU/L (334.5 IU/L); $P>0.05]$.

As expected, untreated IRI rats subjected to 60 minutes of ischemia followed by 24 hours of reperfusion experienced a significant increase in serum ALT levels compared to the Sh-only animals [median (IQR); IRI-only: 2429.5 IU/L (3536.3 IU/L) vs. Sh-only: 59.0 IU/L (38.0 IU/L), $P<0.001]$. Administration of HES significantly decreased serum ALT levels [median (IQR); IRIonly: 2429.5 IU/L (3536.3 IU/L) vs. IRI+HES: 119.0 IU/L (85.5 IU/L), $P=0.001$ ], showing a 20 -fold reduction of IRI-controls'ALT values (Figure 1). Administration of $7.5 \%$ saline also decreased serum ALT levels [median (IQR); IRI-only: 2429.5 IU/L (3536.3 IU/L) vs. IRI+HS: 717 IU/L (4367 IU/L), $P>0.05$ ], however this reduction had no significance (Figure 1).

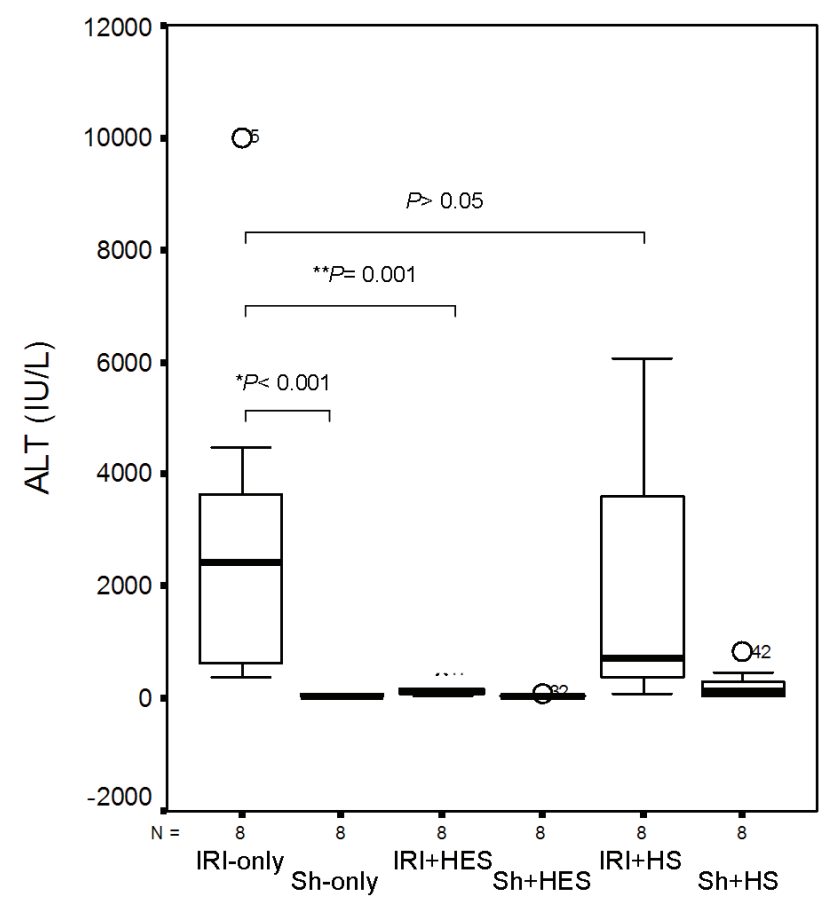

FIGURE 1 - Serum alanine transaminase (ALT) levels. Ischemia/ reperfusion injury to the liver (IRI-only group) significantly enhanced ALT values, compared with the sham control $\left({ }^{*} P<0.001\right)$. HES fluid significantly reduced plasma ALT levels following hepatic IRI (IRI+HES), compared with the untreated IRI (IRI-only) rats $\left({ }^{* *} P=0.001\right)$, contrarily to $7.5 \%$ saline solution (IRI+HS) $(P>0.05)$. Boxplots represent the median and interquartile range values of 8 animals in each group.

\section{Effect of iv HES fluid on hepatocellular injury}

No morphological changes were observed in the livers of sham-operated rats (Figure 2A and $2 \mathrm{~B}$ and 2C), in contrast with the severe and extensive hepatocellular damage observed in untreated IRI rats 24 hours after reperfusion (Figure 2D). This was manifested as extensive areas of coagulative cell necrosis, more than apoptosis. Treatment with HES reduced the extent and severity of hepatic injury in the IRI rats (Figure 2E) and showed a significant decrease in the extent of hepatic cellular necrosis compared with the IRI-only group [Median (IQR); IRI+HES: 0.5\% (5.7\%) vs. IRI-only: $53.9 \%$ (46.7\%), $P<0.01]$, as shown in Figure 3. Furthermore, the percentage of cellular necrosis area was similar between IRI+HES and sham controls [Median (IQR); IRI+HES: $0.5 \%(5.7 \%) v s$. Sham-operated rats: Sh+HES: $0.2 \%$ (0.3\%); Sh+HS 0.5\% (0.9\%); Sh-only: 0.5\% (0.7\%); comparisons between pairs of groups is $P>0.05$ ).

Treatment with $7.5 \%$ saline solution reduced the extent and severity of hepatic injury in the IRI rats (Figure 2F) and showed no significant reduction in the extent of hepatic cellular necrosis compared with the untreated IRI rats [Median (IQR); IRI+HS: $38.5 \%(76.5 \%) v s$. IRI-only: $53.9 \%(46.7 \%), P>0.05$ ], as shown in Figure 3.
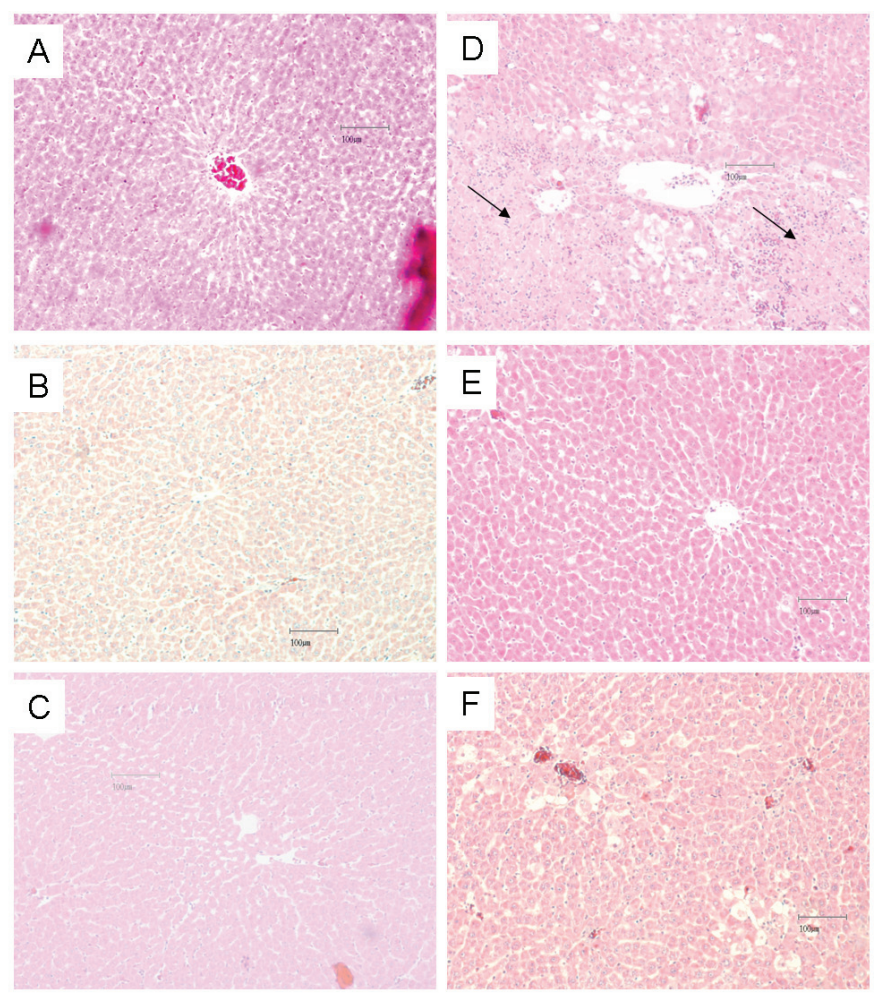

FIGURE 2 - Histopathology of liver. A: Sh-only, B: Sh+HES and C: $\mathrm{Sh}+\mathrm{HS}$ photomicrographs showing normal liver arrangement in sham controls. D: IRI-only photomicrograph showing extensive foci of hepatocellular necrosis indicated by arrows. E: IRI+HES and F: IRI+HS photomicrographs showing less liver derangement than IRI-only. Hematoxylin and eosin stain. The horizontal bar in the pictures represents $100 \mu \mathrm{m}$. 


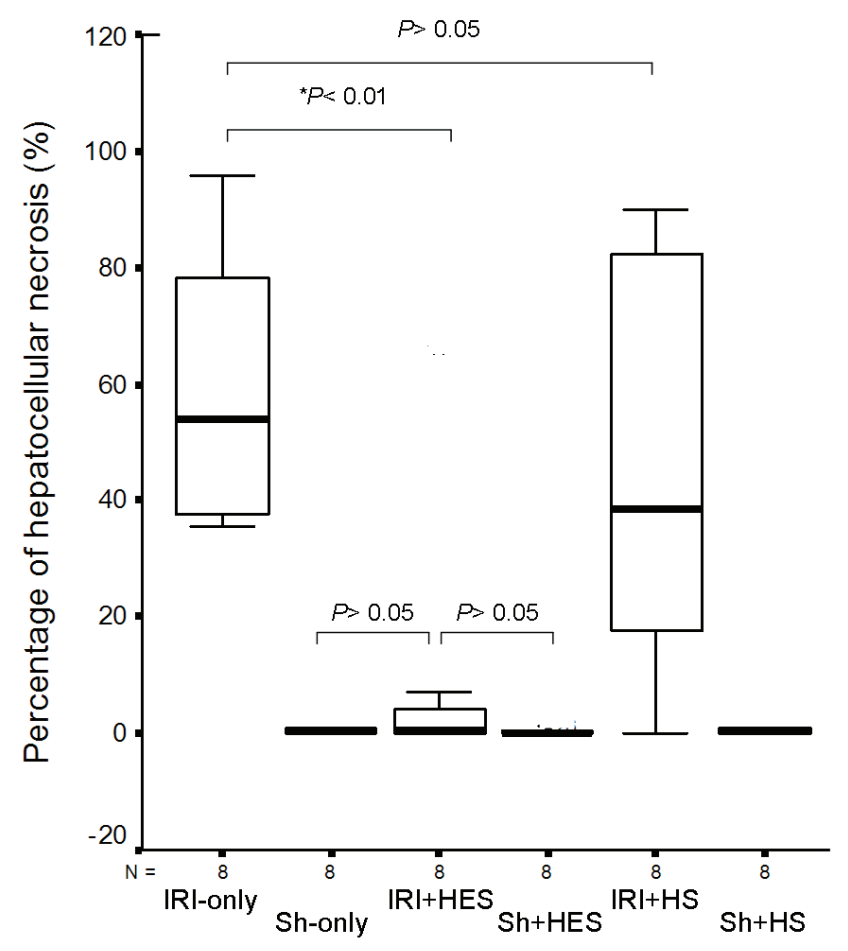

FIGURE 3 - Percentage of hepatocellular necrosis following 60 minutes of ischemia and 24 hours of reperfusion. Treatment with HES showed a significant decrease in the extent of hepatic cellular necrosis in the IRI+HES group, compared with the IRI-only group $\left({ }^{*} P<0.01\right)$. The percentage of cellular necrosis area was similar between IRI-only and IRI+HS $(P>0.05)$ as well as between IRI+HES and the Sham-operated rats $(P>0.05)$. Boxplots represent the median and interquartile range values of 8 animals in each group.

\section{Effect of iv HES fluid in neutrophil infiltration}

The normal (Sh-only, Sh+HES and $\mathrm{Sh}+\mathrm{HS}$ ) rat liver did not exhibit any distinctive evidence of inflammatory cell infiltrate. In the IRI-only animals, intraparenchymal neutrophils were the dominant cells within the necrotic areas, as evidenced by the bright red color of the chloroacetate esterase activity. Consistent with the improvement in the liver necrosis area extension, neutrophil count significantly decreased with HES treatment (IRI+HES: 106 \pm 33.7 vs. IRI-only: $166 \pm 58.8, P<0.05)$, as shown in Figure 4. Regarding treatment with saline, only a slight decrease in the number of liver neutrophils was noted, without statistical significance (IRI+HS: $148 \pm 107.2 v s$. IRI-only: $166 \pm 58.8, P>0.05)$.

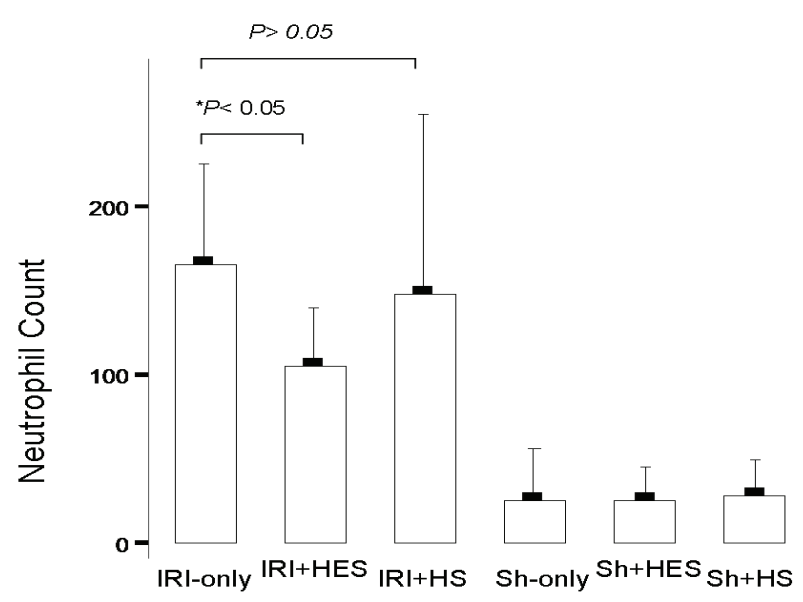

FIGURE 4 - Neutrophil counts within liver tissue, quantified as described in materials and methods (chloroacetate esterase stain, 10 high power fields/section, 8 sections per group). Contrarily to $7.5 \%$ saline solution $(P>0.05)$, HES inhibited hepatic neutrophil infiltration following IRI, showing a significant decrease $\left({ }^{*} P<0.05\right)$ of IRI-only neutrophil count. Values are means $\pm \mathrm{SD}$ of 8 animals per group.

\section{Mortality}

There were no deaths. As expected, all the animals awaked uneventfully and reached the defined end-point (24 hours of reperfusion).

\section{Discussion}

With the use of in vivo rat liver IRI model, this study demonstrates, for the first time, the ability of HES 130/0.4 to attenuate the hepatic damage in the late-phase of liver IRI. Treatment of rats subjected to 60 minutes of ischemia followed by 24 hours of reperfusion with intravenous solution of HES significantly reduced hepatic enzyme elevation and necrosis, with a concomitant reduction in neutrophil sequestration into the ischemic liver, when compared with untreated IRI rats.

The biochemical and histological markers used were the most reflective of hepatic injury in the late phase, having been used in many liver studies ${ }^{9-10}$, and so were chosen to demonstrate the potential protective effect of HES 130/0.4 in this setting; further mechanistic information was obtained by neutrophil staining with Chloroacetate Esterase to reveal a potential anti-inflammatory action, thereby attending to all objectives defined for this study.

Late-phase ischemia/reperfusion injury (IRI) mechanisms are very different from the early-phase ones, as has been widely described $^{1,2}$. Hence, HES 130/0.4 amelioration of the early phase is not necessarily illustrative of the impact of the treatment in the late phase. Studies involving 6\% HES 200/0.62 in skeletal muscle 
ischemia, as referred by Kaplan et al. ${ }^{6}$, showed loss of late effect (24h) on neutrophil adherence even though its presence was found in early phases (30 minutes and 2 hours) of injury ${ }^{11}$.

In this study we focused on the action of HES on neutrophil infiltration, as a measure to assess the mechanism by which the HES fluid attenuates the hepatic late IRI in this model.

It is generally accepted that neutrophils play a critical role in pathogenesis of ischemia/reperfusion injury ${ }^{2}$. In this regard it is pertinent to note that neutrophil activation has been long considered the major effector mechanism in liver IRI and promotes tissue injury ${ }^{2}$. Neutrophils contain abundant cytotoxic factors that can provoke parenchymal cells death, through diffusion of reactive oxygen species and induction of intracellular oxidant stress.

Previous studies have demonstrated benefices of HES solution use, supporting the idea that there are several potential mechanisms through which HES may contribute to organ defense in the context of IRI ${ }^{2,6,8,11-13}$. The mechanism for the protective effect of HES in IRI context is not fully understood, but these starches have been shown to mediate a wide range of biological effects, such as volume expander, antioxidant and anti-inflammatory effects, all of which have been proposed to explain their ability to protect against IRI. For example Kaplan et al. ${ }^{6}$ showed that treatment with HES 257/0.47 in newborn pigs concomitantly attenuated increases in leukocyte adherence and vascular permeability during early reperfusion after global cerebral ischemia. Jüttner et al. ${ }^{7}$ showed that infusion of HES $200 \mathrm{kDa} / 0.5$ in clinically relevant doses may have a beneficial effect on microvascular graft perfusion through the resulting hemodilution and decreased P-selectin expression, with subsequent reduced number of leukocyte-platelet complexes and endothelial adhesion. Lang et al. ${ }^{8}$ found significant attenuation of inflammatory markers, such as interleukins 6 and 8 and soluble intercellular adhesion molecule-1, when HES 130/0.4 was used in patients submitted to major abdominal surgery, compared to a crystalloid-based volume therapy.

Our results are consistent with what is known about benefices of HES fluid therapy in in vivo IRI rat experiments. In a previous study ${ }^{14}$, we showed HES 130/0.4 intravenous solution's ability to attenuate the deleterious effects occurring during the early-phase of liver IRI and we acknowledged the possibility for the HES molecule to play a contributing role in liver IRI attenuation, which has occurred beyond the volume effect. The present study further explains the mechanism of action of late-phase IRI and shows, for the first time, that HES 130/0.4 ameliorates the late-phase response of liver IRI and suppresses neutrophil liver sequestration. Although directly not addressed in this study, the HES dependent diminution of neutrophil infiltration in ischemic liver may be related with its known ability of improving microvascular perfusion and diminishing the leukocyte adherence and vascular permeability during early reperfusion, as was reported by other authors ${ }^{12,13}$.

Our work explores the role of the recent third generation HES fluid therapy in the pathophysiology of hepatic injury. The anti-inflammatory effects of intravenous HES administration are increasingly being appreciated and our findings represent a significant advance in the understanding of the role of the HES fluid therapy protection in in vivo experimental hepatic IRI model. These findings come at a time when there is a need to develop novel preventive approaches to minimize IRI and subsequently reduce morbidity and mortality associated with IRI in humans ${ }^{15}$.

The mechanistic basis of IRI are known to be complex, as probably are complex the mechanisms mediating HES 130/0.4 protection, which are impossible to be fully explained in one single study. This research raises novel questions to be answered in future studies, either in vivo or in vitro in different liver cells.

\section{Conclusion}

Hydroxyethyl starch suppresses inflammatory response and ameliorates the late-phase response of hepatic ischemia/ reperfusion injury.

\section{References}

1. Fondevila C, Busuttil RW, Kupiec-Weglinski JW. Hepatic ischemia/ reperfusion injury - a fresh look. Exp Mol Pathol. 2003;74:86-93.

2. Jaeschke H. Mechanisms of liver injury. II. Mechanisms of neutrophil-induced liver cell injury during hepatic ischemiareperfusion and other acute inflammatory conditions. Am J Physiol Gastrointest Liver Physiol. 2006;290:G1083-8.

3. Piazza O, Scarpati G, Tufano R. Update on transfusion solutions during surgery: review of hydroxyethyl starches 130/0.4. Int J Gen Med. 2010;3:287-95.

4. Langeron O, Doelberg M, Ang ET, Bonnet F, Capdevila X, Coriat P. Voluven, a lower substituted novel hydroxyethyl starch (HES $130 / 0.4$ ), causes fewer effects on coagulation in major orthopedic surgery than HES 200/0.5. Anesth Analg. 2001;92:855-62.

5. Godet G, Lehot JJ, Janvier G, Steib A, De Castro V, Coriat P. Safety of HES 130/0.4 (Voluven(R) in patients with preoperative renal dysfunction undergoing abdominal aortic surgery: a prospective, randomized, controlled, parallel-group multicentre trial. Eur J Anaesthesiol. 2008;25:986-94.

6. Kaplan SS, Park TS, Gonzales ER, Gidday JM. Hydroxyethyl starch reduces leukocyte adherence and vascular injury in the newborn pig cerebral circulation after asphyxia. Stroke. 2000;31:2218-23.

7. Jüttner B, Kuse ER, Elsner HA, Heine J, Jaeger K, Piepenbrock S, Scheinichen D. Differential platelet receptor expression following hydroxyethyl starch infusion in thrombocytopaenic orthotopic liver transplantation recipients. Eur J Anaesthesiol. 2004;21:309-13.

8. Lang K, Suttner S, Boldt J, Kumle B, Nagel D. Volume replacement 
with HES 130/0.4 may reduce the inflammatory response in patients undergoing major abdominal surgery. Can J Anaesth. 2003;50:100916.

9. Serafín A, Roselló-Catafau J, Prats N, Xaus C, Gelpí E, Peralta C. Ischemic preconditioning increases the tolerance of Fatty liver to hepatic ischemia-reperfusion injury in the rat. Am J Pathol. 2002;161:587-601.

10. Hasegawa T, Malle E, Farhood A, Jaeschke H. Generation of hypochlorite-modified proteins by neutrophils during ischemia-reperfusion injury in rat liver: attenuation by ischemic preconditioning. Am J Physiol Gastrointest Liver Physiol. 2005;289:G760-7.

11. Menger MD, Thierjung C, Hammersen F, Messmer K. Dextran vs. hydroxyethylstarch in inhibition of postischemic leukocyte adherence in striated muscle. Circ Shock. 1993;41:248-5.

12. Zikria BA, Subbarao C, Oz MC, Shih ST, McLeod PF, Sachdev R, Freeman HP, Hardy MA. Macromolecules reduce abnormal microvascular permeability in rat limb ischemia/reperfusion injury. Crit Care Med. 1989;17:1306-9.

13. Handrigan MT, Burns AR, Donnachie EM, Bowden RA. Hydroxyethyl starch inhibits neutrophil adhesion and transendothelial migration. Shock. 2005;24:434-9.

14. Catré D, Viana JS, Cabrita AM, Oliveira M, Felizes A, Lopes MF. Hydroxyethyl starch 130/0.4 attenuates early hepatic damage in ischemia/reperfusion injury. Can J Anaesth. 2010;57:439-5.

15. Vollmar B, Menger MD. The hepatic microcirculation: mechanistic contributions and therapeutic targets in liver injury and repair. Physiol Rev. 2009;89:1269-339.

\section{Acknowledgement}

We express our appreciation to Margitta Lebofsky, Research Associate of the University of Kansas Medical Center, Department of Pharmacology, Kansas City, USA, for providing the protocol for cloroacethate staining of neutrophils.

\section{Correspondence:}

Dr. Maria Francelina Lopes

Hospital Pediátrico

Centro Hospitalar e Universitário de Coimbra, EPE

Avenida Afonso Romão, Santo António dos Olivais

3000-602 Coimbra, Portugal

Tel./Fax: 00351239488700 / 00351239717216

mfrancelina@yahoo.com

Received: May 10, 2011

Review: July 14, 2011

Accepted: August 11, 2011

Conflict of interest: none

Financial source: Faculty of Medicine of the University of Coimbra

${ }^{1}$ Research performed at Unit of Experimental Surgery, Faculty of Medicine, University of Coimbra, Portugal. 